\title{
A recurrence relation for the "inv" analogue of $q$-Eulerian polynomials
}

\author{
Chak-On Chow \\ Department of Mathematics and Information Technology, \\ Hong Kong Institute of Education, \\ 10 Lo Ping Road, Tai Po, New Territories, Hong Kong \\ cchow@alum.mit.edu
}

Submitted: Feb 23, 2010; Accepted: Apr 12, 2010; Published: Apr 19, 2010

Mathematics Subject Classifications: 05A05, 05A15

\begin{abstract}
We study in the present work a recurrence relation, which has long been overlooked, for the $q$-Eulerian polynomial $A_{n}^{\text {des,inv }}(t, q)=\sum_{\sigma \in \mathfrak{S}_{n}} t^{\operatorname{des}(\sigma)} q^{\operatorname{inv}(\sigma)}$, where $\operatorname{des}(\sigma)$ and $\operatorname{inv}(\sigma)$ denote, respectively, the descent number and inversion number of $\sigma$ in the symmetric group $\mathfrak{S}_{n}$ of degree $n$. We give an algebraic proof and a combinatorial proof of the recurrence relation.
\end{abstract}

\section{Introduction}

Let $\mathfrak{S}_{n}$ denote the symmetric group of degree $n$. Any element $\sigma$ of $\mathfrak{S}_{n}$ is represented by the word $\sigma_{1} \sigma_{2} \cdots \sigma_{n}$, where $\sigma_{i}=\sigma(i)$ for $i=1,2, \ldots, n$. Two well-studied statistics on $\mathfrak{S}_{n}$ are the descent number and the inversion number defined by

$$
\begin{aligned}
& \operatorname{des}(\sigma):=\sum_{i=1}^{n} \chi\left(\sigma_{i}>\sigma_{i+1}\right), \\
& \operatorname{inv}(\sigma):=\sum_{1 \leqslant i<j \leqslant n} \chi\left(\sigma_{i}>\sigma_{j}\right),
\end{aligned}
$$

respectively, where $\sigma_{n+1}:=0$ and $\chi(P)=1$ or 0 depending on whether the statement $P$ is true or not. It is well-known that des is Eulerian and that inv is Mahonian. The generating function of the Euler-Mahonian pair (des, inv) over $\mathfrak{S}_{n}$ is the following $q$ Eulerian polynomial:

$$
A_{n}^{\mathrm{des}, \operatorname{inv}}(t, q):=\sum_{\sigma \in \mathfrak{S}_{n}} t^{\operatorname{des}(\sigma)} q^{\operatorname{inv}(\sigma)}
$$


It is clear that $A_{n}(t, 1) \equiv A_{n}(t)$, the classical Eulerian polynomial. Let $z$ and $q$ be commuting indeterminates. For $n \geqslant 0$, let $[n]_{q}:=1+q+q^{2}+\cdots+q^{n-1}$ be a $q$-integer, and $[n]_{q} !:=[1]_{q}[2]_{q} \cdots[n]_{q}$ be a $q$-factorial. Define a $q$-exponential function by

$$
e(z ; q):=\sum_{n \geqslant 0} \frac{z^{n}}{[n]_{q} !} .
$$

Stanley [6] proved that

$$
A^{\text {des,inv }}(x, t ; q):=\sum_{n \geqslant 0} A_{n}^{\text {des,inv }}(t, q) \frac{x^{n}}{[n]_{q} !}=\frac{1-t}{1-t e(x(1-t) ; q)} .
$$

Alternate proofs of (1) have also been given by Garsia [4] and Gessel [5]. Désarménien and Foata [2] observed that the right side of (1) is precisely

$$
\left(1-t \sum_{n \geqslant 1}(1-t)^{n-1} \frac{x^{n}}{[n]_{q} !}\right)^{-1},
$$

and from which they obtained a "semi" $q$-recurrence relation for $A_{n}^{\text {des,inv }}(t, q)$, namely,

$$
A_{n}^{\mathrm{des}, \text { inv }}(t, q)=t(1-t)^{n-1}+\sum_{1 \leqslant i \leqslant n-1}\left[\begin{array}{c}
n \\
i
\end{array}\right]_{q} A_{i}^{\mathrm{des}, \operatorname{inv}}(t, q) t(1-t)^{n-1-i} .
$$

The above $q$-recurrence relation is "semi" in the sense that the summands on the right involve two factors one of which depends on $q$ whereas the other does not. We shall establish in the present note that a "fully" $q$-recurrence relation for $A_{n}^{\text {des,inv }}(t, q)$ exists such that both factors of the summands depend on $q$ (see Theorem 2.2 below). In the next section, we derive this recurrence relation algebraically. In the final section, we give a combinatorial proof of this recurrence relation.

\section{The recurrence relation}

We derive in the present section the recurrence relation by algebraic means.

Let $\mathbb{Q}$ denote, as customary, the set of rational numbers. Let $x$ be an indeterminate, $\mathbb{Q}[x]$ be the ring of polynomials in $x$ over $\mathbb{Q}$, and $\mathbb{Q}[[x]]$ the ring of formal power series in $x$ over $\mathbb{Q}$. We introduce an Eulerian differential operator $\delta_{x}$ in $x$ by

$$
\delta_{x}(f(x))=\frac{f(q x)-f(x)}{q x-x},
$$

for any $f(x) \in \mathbb{Q}[q][[x]]$ in the ring of formal power series in $x$ over $\mathbb{Q}[q]$. It is easy to see that

$$
\delta_{x}\left(x^{n}\right)=[n]_{q} x^{n-1},
$$

so that as $q \rightarrow 1, \delta_{x}\left(x^{n}\right) \rightarrow n x^{n-1}$, the usual derivative of $x^{n}$. See [1] for further properties of $\delta_{x}$. 
LEMMA 2.1. We have $\delta_{x}(e(x(1-t) ; q)=(1-t) e(x(1-t) ; q)$.

Proof. This follows from

$$
\begin{aligned}
\delta_{x}(e(x(1-t) ; q) & =\frac{e(q x(1-t) ; q)-e(x(1-t) ; q)}{(q-1) x} \\
& =\sum_{n \geqslant 0} \frac{q^{n} x^{n}(1-t)^{n}-x^{n}(1-t)^{n}}{(q-1) x[n]_{q} !} \\
& =\sum_{n \geqslant 1} \frac{x^{n-1}(1-t)^{n}}{[n-1]_{q} !} \\
& =(1-t) e(x(1-t) ; q) .
\end{aligned}
$$

Theorem 2.2. For $n \geqslant 1, A_{n}^{\text {des,inv }}(t, q)$ satisfies

$$
A_{n+1}^{\text {des,inv }}(t, q)=\left(1+t q^{n}\right) A_{n}^{\text {des,inv }}(t, q)+\sum_{k=1}^{n-1}\left[\begin{array}{l}
n \\
k
\end{array}\right]_{q} q^{k} A_{n-k}^{\text {des,inv }}(t, q) A_{k}^{\text {des,inv }}(t, q) \text {. }
$$

Proof. From (1) we have that

$$
t e(x(1-t) ; q)=\frac{A^{\mathrm{des}, \operatorname{inv}}(x, t ; q)-(1-t)}{A^{\operatorname{des}, \operatorname{inv}}(x, t ; q)} .
$$

Applying $\delta_{x}$ to both sides of (1), and using Lemma 2.1, (1) and (3), we have

$$
\begin{aligned}
\sum_{n \geqslant 0} A_{n+1}^{\text {des,inv }}(t, q) \frac{x^{n}}{[n]_{q} !} & =\frac{(1-t)}{(q-1) x}\left(\frac{1}{1-t e(q x(1-t) ; q)}-\frac{1}{1-t e(x(1-t) ; q)}\right) \\
& =\frac{t(1-t) \delta_{x}(e(x(1-t) ; q)}{[1-t e(x(1-t) ; q)][1-t e(q x(1-t) ; q)]} \\
& =\frac{t(1-t)^{2} e(x(1-t) ; q)}{[1-t e(x(1-t) ; q)][1-t e(q x(1-t) ; q)]} \\
& =\left[A^{\text {des,inv }}(x, t ; q)-(1-t)\right] A^{\text {des,inv }}(q x, t ; q) .
\end{aligned}
$$

Extracting the coefficients of $x^{n}$, we finally have

$$
\begin{aligned}
A_{n+1}^{\text {des,inv }}(t, q) & =\sum_{k=0}^{n}\left[\begin{array}{l}
n \\
k
\end{array}\right]_{q} q^{k} A_{n-k}^{\text {des,inv }}(t, q) A_{k}^{\text {des,inv }}(t, q)-(1-t) q^{n} A_{n}^{\text {des,inv }}(t, q) \\
& =\left(1+t q^{n}\right) A_{n}^{\text {des,inv }}(t, q)+\sum_{k=1}^{n-1}\left[\begin{array}{l}
n \\
k
\end{array}\right]_{q} q^{k} A_{n-k}^{\text {des,inv }}(t, q) A_{k}^{\text {des,inv }}(t, q) .
\end{aligned}
$$


The identity (2) is a $q$-analogue of the following convolution-type recurrence [3, p. 70]

$$
A_{n+1}(t)=(1+t) A_{n}(t)+\sum_{k=1}^{n-1}\left(\begin{array}{l}
n \\
k
\end{array}\right) A_{n-k}(t) A_{k}(t),
$$

satisfied by the classical Eulerian polynomials $A_{n}(t):=\sum_{\sigma \in \mathfrak{S}_{n}} t^{\operatorname{des}(\sigma)}$.

\section{A combinatorial proof}

We give a combinatorial proof of Theorem 2.2 in the present section.

Recall that elements of $\mathfrak{S}_{n+1}$ can be obtained by inserting $n+1$ to elements of $\mathfrak{S}_{n}$. Let $\sigma=\sigma_{1} \cdots \sigma_{n} \in \mathfrak{S}_{n}$. Denote by $\sigma_{+k}=\sigma_{1} \cdots \sigma_{k}(n+1) \sigma_{k+1} \cdots \sigma_{n}, 0 \leqslant k \leqslant n$. It is easy to see that

$$
\begin{aligned}
& \operatorname{des}\left(\sigma_{+0}\right)=\operatorname{des}(\sigma)+1, \quad \operatorname{inv}\left(\sigma_{+0}\right)=\operatorname{inv}(\sigma)+n, \\
& \operatorname{des}\left(\sigma_{+n}\right)=\operatorname{des}(\sigma), \quad \operatorname{inv}\left(\sigma_{+n}\right)=\operatorname{inv}(\sigma),
\end{aligned}
$$

and for $1 \leqslant k \leqslant n-1$,

$$
\begin{aligned}
\operatorname{des}\left(\sigma_{+k}\right)= & \operatorname{des}\left(\sigma_{1} \cdots \sigma_{k}\right)+\operatorname{des}\left(\sigma_{k+1} \cdots \sigma_{n}\right), \\
\operatorname{inv}\left(\sigma_{+k}\right)= & \operatorname{inv}\left(\sigma_{1} \cdots \sigma_{k}\right)+\operatorname{inv}\left(\sigma_{k+1} \cdots \sigma_{n}\right) \\
& +n-k+\#\left\{(r, s): \sigma_{r}>\sigma_{s}, 1 \leqslant r \leqslant k, k+1 \leqslant s \leqslant n\right\} .
\end{aligned}
$$

Let $S=\left\{\sigma_{1}, \ldots, \sigma_{k}\right\}$. Then the partial permutations $\sigma_{1} \cdots \sigma_{k} \in \mathfrak{S}(S)$ and $\sigma_{k+1} \cdots \sigma_{n} \in$ $\mathfrak{S}([n] \backslash S)$, where $\mathfrak{S}(S)$ denotes the group of permutations of the set $S$. It is clear that the product $\mathfrak{S}(S) \times \mathfrak{S}([n] \backslash S)$ is a subgroup of $\mathfrak{S}_{n}$ isomorphic to $\mathfrak{S}_{k} \times \mathfrak{S}_{n-k}$. Also, the quotient $\mathfrak{S}_{n} /\left(\mathfrak{S}_{k} \times \mathfrak{S}_{n-k}\right) \cong\left(\begin{array}{c}{[n]} \\ k\end{array}\right)$ (see [8, p. 351]), where $\left(\begin{array}{c}{[n]} \\ k\end{array}\right)$ denotes the set of all $k$-subsets of $[n]$, which is in bijective correspondence with the set of multipermutations $\mathfrak{S}\left(\left\{1^{k}, 2^{n-k}\right\}\right)$ of the multiset $\left\{1^{k}, 2^{n-k}\right\}$ consisting of $k$ copies of 1 's and $n-k$ copies of 2's.

Define a multipermutation $w=w_{1} w_{2} \cdots w_{n} \in \mathfrak{S}\left(\left\{1^{k}, 2^{n-k}\right\}\right)$ by

$$
w_{i}= \begin{cases}1 & \text { if } i \in S=\left\{\sigma_{1}, \ldots, \sigma_{k}\right\} \\ 2 & \text { if } i \in[n] \backslash S=\left\{\sigma_{k+1}, \ldots, \sigma_{n}\right\}\end{cases}
$$

Let $1 \leqslant i<j \leqslant n$. It is clear that $(i, j)$ is an inversion of $w$ if and only if $i=\sigma_{s}, j=\sigma_{r}$ for some $1 \leqslant r \leqslant k, k+1 \leqslant s \leqslant n$ and $\sigma_{r}>\sigma_{s}$, so that

$$
\#\left\{(r, s): \sigma_{r}>\sigma_{s}, 1 \leqslant r \leqslant k, k+1 \leqslant s \leqslant n\right\}=\operatorname{inv}(w) .
$$

As $S$ ranges over $\left(\begin{array}{c}{[n]} \\ k\end{array}\right), w$ so defined ranges over $\mathfrak{S}\left(\left\{1^{k}, 2^{n-k}\right\}\right)$. Putting pieces together and using the fact [7, Proposition 1.3.17] that

$$
\sum_{w \in \mathfrak{S}\left(\left\{1^{k}, 2^{n-k}\right\}\right)} q^{\operatorname{inv}(w)}=\left[\begin{array}{l}
n \\
k
\end{array}\right]_{q},
$$


we have

$$
\begin{aligned}
& A_{n+1}^{\mathrm{des}, \text { inv }}(t, q) \\
& =\sum_{k=0}^{n} \sum_{\sigma \in \mathfrak{S}_{n}} t^{\operatorname{des}\left(\sigma_{+k}\right)} q^{\operatorname{inv}\left(\sigma_{+k}\right)} \\
& =\left(1+t q^{n}\right) A_{n}^{\mathrm{des}, \mathrm{inv}}(t, q) \\
& +\sum_{k=1}^{n-1} \sum_{\begin{array}{c}
\sigma_{1} \cdots \sigma_{k} \in \mathfrak{S}_{k} \\
\sigma_{k+1} \cdots \sigma_{n} \in \mathfrak{S}_{n-k} \\
w \in \mathfrak{S}\left(\left\{1^{k}, 2^{-k}\right\}\right)
\end{array}} t^{\operatorname{des}\left(\sigma_{1} \cdots \sigma_{k}\right)+\operatorname{des}\left(\sigma_{k+1} \cdots \sigma_{n}\right)} q^{\operatorname{inv}\left(\sigma_{1} \cdots \sigma_{k}\right)+\operatorname{inv}\left(\sigma_{k+1} \cdots \sigma_{n}\right)+n-k+\operatorname{inv}(w)} \\
& =\left(1+t q^{n}\right) A_{n}^{\mathrm{des}, \operatorname{inv}}(t, q)+\sum_{k=1}^{n-1} q^{n-k} \sum_{w \in \mathfrak{S}\left(\left\{1^{k}, 2^{n-k}\right\}\right)} q^{\operatorname{inv}(w)} \sum_{\tau \in \mathfrak{S}_{k}} t^{\operatorname{des}(\tau)} q^{\operatorname{inv}(\tau)} \sum_{\pi \in \mathfrak{S}_{n-k}} t^{\operatorname{des}(\pi)} q^{\operatorname{inv}(\pi)} \\
& =\left(1+t q^{n}\right) A_{n}^{\mathrm{des}, \mathrm{inv}}(t, q)+\sum_{k=1}^{n-1} q^{n-k}\left[\begin{array}{c}
n \\
k
\end{array}\right]_{q} A_{k}^{\mathrm{des}, \text { inv }}(t, q) A_{n-k}^{\mathrm{des}, \text { inv }}(t, q),
\end{aligned}
$$

which is equivalent to (2) (by virtue of the symmetry of the $q$-binomial coefficient).

\section{References}

[1] G.E. Andrews, On the foundations of combinatorial theory V, Eulerian differential operators, Stud. Appl. Math. 50 (1971), 345-375.

[2] J. Désarménien and D. Foata, Signed Eulerian numbers, Discrete Math. 99 (1992), 49-58.

[3] D. Foata and M.-P. Schützenberger, Théorie géométrique des polynômes Eulériens, Lecture Notes in Mathematics, vol. 138, Springer-Verlag, Berlin-New York, 1970.

[4] A.M. Garsia, On the "maj" and "inv" analogues of Eulerian polynomials, Linear and Multilinear Algebra 8 (1979), 21-34.

[5] I.M. Gessel, Generating Functions and Enumeration of Sequences, Ph.D. thesis, Massachusetts Institute of Technology, June 1977.

[6] R.P. Stanley, Binomial posets, Möbius inversion and permutation enumeration, J. Combin. Theory Ser. A 20 (1976), 336-356.

[7] R.P. Stanley, Enumerative Combinatorics, vol. 1, Cambridge University Press, Cambridge, 1997.

[8] R.P. Stanley, Enumerative Combinatorics, vol. 2, Cambridge University Press, Cambridge, 1999. 